\title{
Electrochemical Oxidation of D-Sorbitol and D-Manitol on Platinum Monocrystalline Surfaces
}

\author{
L. Proença *, A.J. Silva, M.I.S. Lopes, I.T.E. Fonseca, \\ A. Rodes ${ }^{\text {a) }}$, A. Aldaz ${ }^{\text {a) }}$ \\ CECUL, Departamento de Quimica e Bioquímica, Faculdade de Ciências da Universidade de \\ Lisboa, Edifício C8, Campo Grande, 1749-016 Lisboa, Portugal. \\ a) Departamento de Química Física, Universidad de Alicante, Apartado 99, \\ E-03080 Alicante, España
}

Received 10 January 2003; accepted 6 November 2003

\begin{abstract}
The electrochemical oxidation of D-sorbitol and D-mannitol was studied on two sets of stepped platinum single-crystal surfaces vicinal to the basal plane (111), as well as on a set of surfaces with monoatomic steps vicinal to the surface $\operatorname{Pt}(100)$. The aim of this study, conducted by cyclic voltammetry, was to check the influence of the crystalline surface structure (regarding terrace and step orientations) on the electrochemical oxidation process of the two polyols in platinum.

The electrochemical oxidation reactions of D-sorbitol and D-mannitol were shown to be markedly influenced by the symmetry of the bidimensional domains as well as by the distribution of the orientated defects (monoatomic steps) on the platinum surfaces. The introduction of orientated defects leads to a reduction on peak current densities along with an increase in activity at lower potentials. The terrace width is clearly decisive to the oxidation process on the set of surfaces belonging to the series $\operatorname{Pt}(\mathrm{S})[(n-1)(111) \times$ (110)].
\end{abstract}

Keywords: Electrochemical oxidation, D-sorbitol, D-manitol, platinum, single-crystal surfaces, monoato-mic steps.

\section{Resumo}

A oxidação electroquímica do D-sorbitol e D-manitol foi estudada para dois conjuntos de superfícies monocristalinas de platina, com degraus monoatómicos, vicinais ao plano basal (111) e ainda para um conjunto de superfícies com degraus monoatómicos

\footnotetext{
*Corresponding author. E-mail address: lpro@fc.ul.pt
} 
vicinais à superfície $\mathrm{Pt}(100)$. Este estudo, por voltametria cíclica, teve por objectivo obter informação sobre a influência da geometria de sítios superficiais (tanto da orientação dos degraus quanto da dos terraços) no processo de oxidação electroquímica dos dois polióis em platina.

A reacção de oxidação electroquímica do D-sorbitol e D-manitol é sensível à simetria dos domínios bidimensionais bem como à distribuição dos defeitos orientados (degraus monoatómicos) nas superfícies de platina. A introdução de defeitos ordenados leva a uma redução geral das densidades de corrente de pico a par de um aumento de actividade aos potenciais menos positivos. A largura dos terraços é claramente decisiva para o processo de oxidação nas superfícies pertencentes à série $\operatorname{Pt}(\mathrm{S})[(n-1)(111) \times$ (110)].

PalavrasChave: Oxidação electroquímica, D-sorbitol, D-manitol, platina, superficies monocristalinas, degraus monoatómicos.

\section{Introdução}

Os planos de eléctrodos monocristalinos metálicos são as superfícies mais simples existentes. São as menos complexas e menos pormenorizadas, e a esse nível, as mais perfeitas. Talvez, mais que perfeitas, seja mais acertado chamarlhes úteis. A contribuição das superfícies monocristalinas bem definidas é cada vez mais importante no domínio da investigação em electrocatálise e química das superfícies [1-3].

O efeito catalítico de um metal é, sobretudo, resultado da estrutura electrónica. A consciência de que outra diferença estrutural, de natureza geométrica, é cataliticamente importante, corresponde à possibilidade de se poder avaliar os processos de eléctrodo de forma mais completa. Sempre enfatizando, todavia, que os factores electrónicos e de estrutura geométrica não são inteiramente separáveis nas suas contribuições para o processo electroquímico global, é possível, ainda assim, proceder ao estudo individual dos efeitos da estrutura geométrica.

Para este efeito, são possíveis várias modificações estruturais. Talvez a possibilidade mais interessante seja a introdução controlada, à escala molecular, de defeitos na superfície. Sem que a ordem bidimensional de longo alcance seja perdida, é igualmente possível, como para as superfícies de baixo índice de Miller, preparar superfícies ordenadas de elevados índices de Miller com um grande grau de precisão. 
Os defeitos ordenados, no caso - fileiras de degraus monoatómicos, são uma aproximação (semi)ideal à irregularidade na superfície de um policristal. Mas a sua relevância é superior à de uma mera aproximação. Mais que sondas para explicar um comportamento, também revelam comportamentos, em si, novos e por explicar.

Num monocristal conhece-se com precisão o que não é possível conhecer de forma sistematizada noutras superfícies: a coordenação dos átomos superficiais, as distâncias interatómicas, a distância entre os sítios de adsorção, e as energias superficiais. Os estudos realizados com planos monocristalinos, desde o trabalho pioneiro de Clavilier et al. [4], têm contribuído para o estudo sob uma nova perspectiva de processos electroquímicos à superfície do eléctrodo, dando significado e importância fundamental à estrutura superficial.

As superfícies monocristalinas bem definidas foram aplicadas desde finais do século passado na investigação do comportamento electroquímico de pequenas moléculas orgânicas modelares [1]. Presentemente, e cada vez mais, maiores e mais complexas moléculas são analisadas, por exemplo moléculas orgânicas com vários grupos funcionais. $\mathrm{O}$ D-sorbitol e D-manitol são dois compostos hidroxilados (polióis) com uma cadeia carbonada de seis átomos de carbono, estruturalmente semelhantes (estereo-isómeros) e que diferem apenas na posição de um grupo $\mathrm{OH}$.

Este estudo, por voltametria cíclica, refere-se à oxidação electroquímica do Dsorbitol e D-manitol em superfícies monocristalinas de platina, com degraus monoatómicos.

\section{Procedimento Experimental}

Os eléctrodos de platina foram preparados a partir de fios de platina como no método desenvolvido por Clavilier et al. [5]. Nestas experiências voltamétricas utilizaram-se eléctrodos com diâmetros da ordem de $2 \mathrm{~mm}$. Antes de cada experiência, superfícies de platina limpas e bem ordenadas foram preparadas por anelamento à chama e arrefecimento numa atmosfera de árgon e hidrogénio [6]. Depois deste procedimento, durante a transferência para a célula electroquímica, 
as superfícies dos eléctrodos foram protegidas com uma gota de água ultrapura em equilíbrio com ambos os gases.

A água ultrapura utilizada, tanto na lavagem do material como na preparação de soluções, foi purificada através de um sistema Millipore ${ }^{\circledR}$ (Milli-Q), alimentado por água previamente destilada e desionizada.

As soluções foram preparadas a partir de reagentes de elevado grau de pureza, atestada pela marca comercial: $\mathrm{HClO}_{4}$ (Merck, "Suprapur ${ }^{\circledR}$ "), D-manitol (Aldrich-Chemie +99\%) e D-sorbitol (Merck “para fins bioquímicos”). Antes de cada experiência as soluções são desoxigenadas por borbulhamento com árgon (N50, L’Air Liquide), sempre sob agitação mecânica. No decorrer das experiências foi mantida uma corrente de árgon sobre a solução. Todas as experiências foram efectuadas à temperatura ambiente.

Os potenciais de eléctrodo foram medidos em relação a um eléctrodo reversível de hidrogénio (RHE) colocado na mesma solução e são referidos na escala RHE.

\section{Resultados e Discussão}

\section{Superfícies de baixo índice de Miller, Pt(hkl)}

As respostas voltamétricas correspondentes à oxidação electroquímica do Dsorbitol e D-manitol, em superfícies monocristalinas de platina com orientação (100), (110) e (111), mostram que a densidade de corrente máxima diminui com o aumento do número de varrimentos de potencial, indicando a desactivação das superfícies $[7,8]$.

Na Tabela 1 indicam-se as densidades máximas de corrente durante o primeiro e segundo varrimentos de potencial, a uma velocidade de $50 \mathrm{mV} \mathrm{s}^{-1}$, e ainda, os potenciais de pico e percentagens de desactivação (calculadas para a redução de densidade de corrente, do primeiro ciclo para o segundo, no pico principal de oxidação).

Conquanto se atenda apenas às densidades de corrente, a superfície mais activa é a $\operatorname{Pt}(100)$, embora o pico principal de oxidação ocorra a um potencial mais elevado (0.75 $\mathrm{V}$ para o caso do D-sorbitol e $0.74 \mathrm{~V}$ para o D-manitol) que 
noutras superfícies. Por outro lado, a superfície $\operatorname{Pt}(110)$ é a menos activa e a mais envenenável superfície dos três planos basais de platina. A desactivação da $\operatorname{Pt}(100)$ e da $\operatorname{Pt}(111)$ é muito similar $(\sim 40 \%)$.

Em termos absolutos, maiores densidades de corrente são obtidas para a oxidação do D-sorbitol.

Tabela 1. Valores de densidades de corrente de pico máximas (para o primeiro e segundo varrimento), potenciais de pico e desactivação das superfícies $\operatorname{Pt}(h k l)$, durante o processo de oxidação do D-sorbitol e D-manitol.

\begin{tabular}{|c|c|c|c|c|}
\cline { 2 - 5 } \multicolumn{1}{c|}{} & \multicolumn{4}{c|}{ D-sorbitol } \\
\cline { 2 - 5 } \multicolumn{1}{c|}{} & $j_{\mathrm{p}}{ }^{1}\left(\mathrm{~mA} \mathrm{~cm}^{-2}\right)$ & $j_{\mathrm{p}}{ }^{2}\left(\mathrm{~mA} \mathrm{~cm}^{-2}\right)$ & $E_{\mathrm{p}}(\mathrm{V} / \mathrm{RHE})$ & $\begin{array}{c}\text { Desactivação } \\
(\%)\end{array}$ \\
\hline $\operatorname{Pt}(\mathbf{1 1 1})$ & 2.36 & 1.49 & 0.70 & 37 \\
\hline $\operatorname{Pt}(\mathbf{1 1 0})$ & 1.00 & 0.38 & 0.68 & 62 \\
\hline $\operatorname{Pt}(\mathbf{1 0 0})$ & 3.82 & 2.33 & 0.75 & 39 \\
\hline
\end{tabular}

\begin{tabular}{|c|c|c|c|c|}
\cline { 2 - 5 } \multicolumn{1}{c|}{} & \multicolumn{4}{c|}{ D-manitol } \\
\cline { 2 - 5 } \multicolumn{1}{c|}{} & $j_{\mathrm{p}}{ }^{1}\left(\mathrm{~mA} \mathrm{~cm}^{-2}\right)$ & $j_{\mathrm{p}}{ }^{2}\left(\mathrm{~mA} \mathrm{~cm}^{-2}\right)$ & $E_{\mathrm{p}}(\mathrm{V} / \mathrm{RHE})$ & $\begin{array}{c}\text { Desactivação } \\
(\%)\end{array}$ \\
\hline $\operatorname{Pt}(\mathbf{1 1 1})$ & 1.75 & 1.00 & 0.71 & 43 \\
\hline $\operatorname{Pt}(\mathbf{1 1 0})$ & 0.91 & 0.34 & 0.66 & 73 \\
\hline $\operatorname{Pt}(\mathbf{1 0 0})$ & 2.05 & 1.18 & 0.74 & 42 \\
\hline
\end{tabular}

\section{Superficies monocristalinas com degraus monoatómicos}

As superfícies cristalinas, vizinhas dos planos cristalográficos de orientação basal, podem ser decompostas, à escala atómica, em terraços, degraus e defeitos, cada um deles contendo sítios activos com uma simetria específica.

Para além dos planos cristalográficos de orientação (100), (110) e (111), este estudo incidiu especialmente sobre superfícies monocristalinas com degraus monoatómicos. Tal foi levado a cabo com o intuito de analisar o efeito da presença de heterogeneidades (degraus) nas superfícies de orientação basal sobre os processos de oxidação electroquímica do D-sorbitol e D-manitol.

A análise de resultados electroquímicos anteriores [9, 10] revela que estas superfícies regularmente modificadas com degraus podem ser utilizadas em 
estudos na interface eléctrodo|solução se as amostras são preparadas por anelamento à chama e arrefecimento em condições experimentais adequadas.

Em função dos resultados anteriormente obtidos, o estudo foi centrado em torno do plano (111), já que a resposta voltamétrica obtida com esta superfície mostra a presença de uma corrente de oxidação significativa numa vasta região de potencial.

Assim, utilizaram-se superfícies, vicinais ao plano (111), constituídas por degraus monoatómicos e terraços de orientação (111) de dimensão variável. Estas superfícies pertencem às séries cristalográficas que podem ser designadas por $\operatorname{Pt}(\mathrm{S})[n(111) \times(111)]$ e $\operatorname{Pt}(\mathrm{S})[n(111) \times(100)]$, i.e., são superfícies com terraços de orientação (111) e degraus monoatómicos de orientação (111) e (100), respectivamente.

Por último, são apresentados resultados complementares, obtidos para três superfícies pertencentes à série $\operatorname{Pt}(\mathrm{S})[n(100) \times(111)]$, onde se analisa o efeito da dimensão dos terraços de orientação (100) sobre os processos de oxidação electroquímica em estudo.

$\mathrm{Na}$ notação $\operatorname{Pt}(\mathrm{S})\left[n(h k l) \times\left(h^{\prime} k^{\prime} l^{\prime}\right)\right], n$ representa o número de filas densas de átomos nos terraços com estrutura $(1 \times 1)$, separados por degraus monoatómicos cuja densidade, dada pelo modelo de esferas rígidas, é $1 /(n-2 / 3)$ no caso de degraus de orientação (110) e 1/(n-1/3) no caso de degraus de orientação (100).

\section{Série Pt(S) [n (111) × (111)]}

As superfícies pertencentes a esta série, que podem ser designadas por $\operatorname{Pt}(n, n$, n-2), como referido anteriormente, têm a característica de possuir degraus monoatómicos com orientação (111) na junção dos terraços de orientação (111). Como os picos de adsorção de hidrogénio correspondentes aos degraus de orientação (111) no electrólito de suporte são semelhantes aos obtidos com o plano de orientação basal (110) [9], esta série pode ser representada mais adequadamente pelas suas propriedades electroquímicas como $\operatorname{Pt}(\mathrm{S})[(n-1)(111)$ $\times(110)]$. 
Na Fig. 1 estão apresentados os perfis voltamétricos da oxidação electroquímica do D-sorbitol em superfícies monocristalinas do tipo $\operatorname{Pt}(n, n, n-2)$. A dimensão dos terraços foi variada entre 3 e 10 fiadas atómicas. Para estes voltamogramas, a principal região de oxidação situa-se entre 0.3 e $0.8 \mathrm{~V}$. A forma geral dos voltamogramas aproxima-se da obtida no voltamograma registado com a superfície de orientação basal (110) [7].
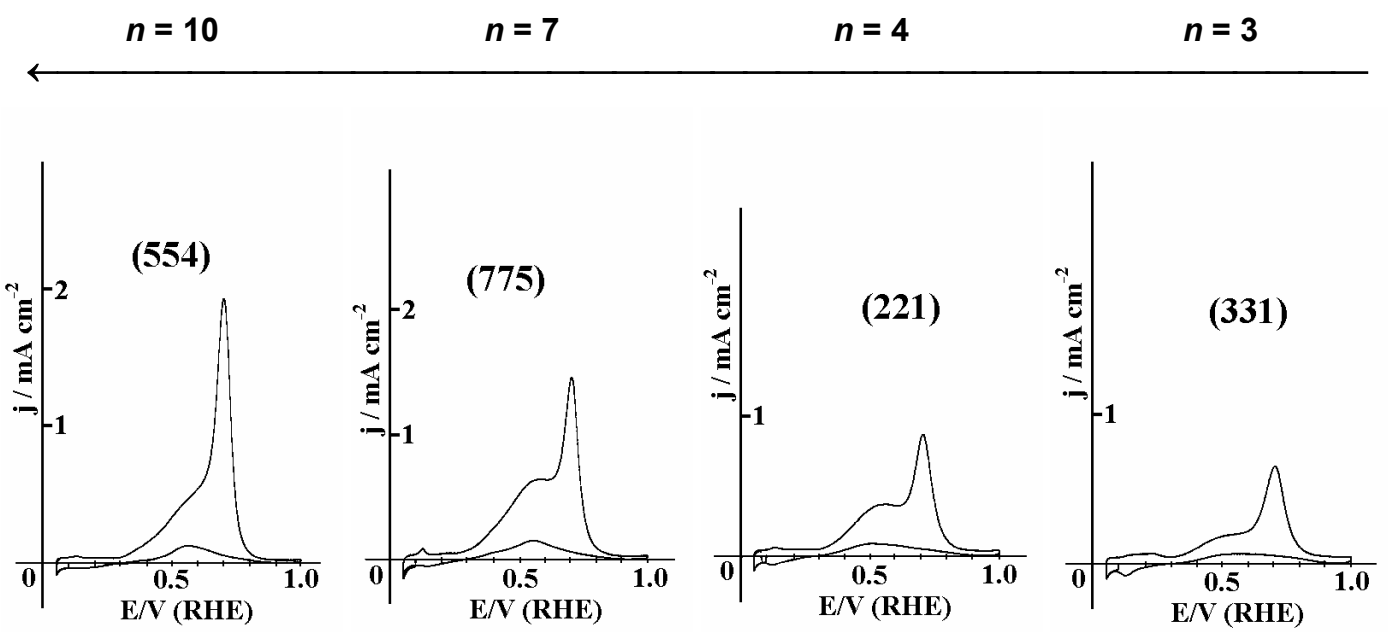

Figura 1. Perfis voltamétricos da oxidação do D-sorbitol $\left(10^{-2} \mathrm{~mol} \mathrm{dm}^{-3}\right)$ em superfícies monocristalinas, com degraus monoatómicos, pertencentes à série $\operatorname{Pt}(\mathrm{S})[(n-1)(111) \times$ (110)], em $\mathrm{HClO}_{4} 0.1 \mathrm{~mol} \mathrm{dm}^{-3} ; v=50 \mathrm{mV} \mathrm{s}^{-1}$.

Os perfis voltamétricos anódicos para a oxidação de D-manitol nas superfícies com degraus monoatómicos, do tipo $\mathrm{Pt}(n, n, n-2)$, em soluções de $\mathrm{HClO}_{4} 0.1$ mol $\mathrm{dm}^{-3}$, são apresentados na Fig. 2. Fez-se igualmente variar a largura dos terraços de 3 a 10 fiadas atómicas. A título comparativo é apresentada a resposta voltamétrica da superfície Pt(111).

Em todas as superfícies com degraus pertencentes a esta série o princípio do pico de oxidação é similar, embora gradualmente se desloque para potenciais menos positivos com a introdução de degraus orientados (110), sugerindo que o mesmo processo de oxidação está envolvido em todas as superfícies de platina, indistintamente. A cobertura superficial, parece ser, todavia, fortemente influenciada pela distribuição geral dos degraus: nos terraços mais largos há uma densidade de corrente de oxidação maior. 
Pode ser notado que a desadsorção do hidrogénio ocorre para um potencial mais baixo em domínios (110) que para domínios com orientação de simetria (111). A introdução de degraus (110) deve, então, ser responsável pelo desvio, em direcção a potenciais mais negativos, do potencial de princípio de pico para o pico principal nesta superfície. Um efeito similar ao exposto, foi já relatado para o processo de oxidação electroquímica da glucose em superfícies de platina com degraus $[11,12]$.

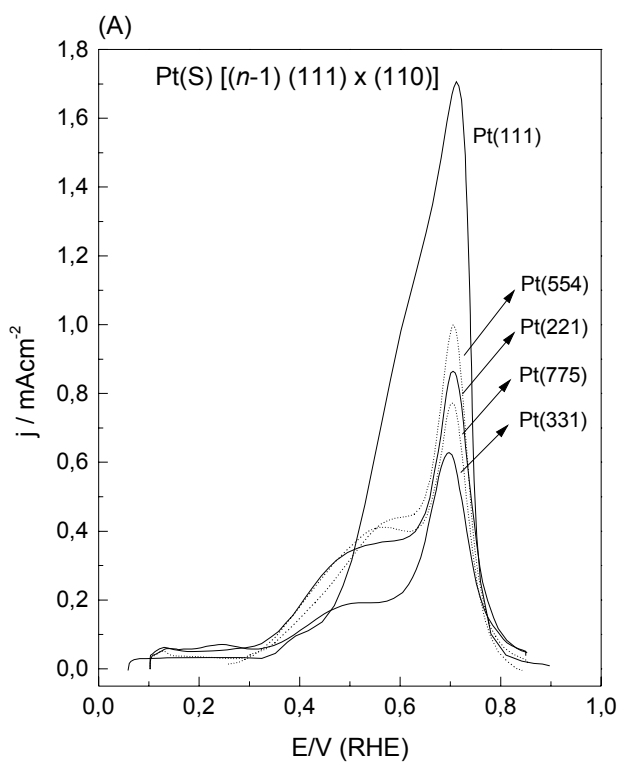

Figura 2. Perfis voltamétricos da oxidação electroquímica do D-manitol $\left(10^{-2} \mathrm{~mol} \mathrm{dm}^{-3}\right)$ em superfícies monocristalinas, com degraus monoatómicos, do tipo $\operatorname{Pt}(\mathrm{S})[(n-1)(111)$ $\times(110)], \mathrm{em} \mathrm{HClO}_{4} 0.1 \mathrm{~mol} \mathrm{dm}^{-3} ; v=50 \mathrm{mV} \mathrm{s}^{-1}$.

Na série inteira a introdução ordenada de degraus leva a uma modificação da desactivação interfacial. Das superfícies com degraus, aquelas onde os terraços de orientação (111) são mais estreitos revelam a maior desactivação percentual. Então, não apenas a cobertura por intermediários, mas ainda a rapidez da desactivação superficial, são modificadas por degraus (110). Como já foi notado para a $\operatorname{Pt}(111)$ [7, 8], a actividade à superfície está confinada à região de potencial abaixo do maior potencial dos estados de adsorção do hidrogénio em meio ácido. Os picos voltamétricos principais decrescem até ao bloqueio total da actividade nesta região de potencial. 
Na Fig. 3 representa-se graficamente a variação do valor de densidade de corrente máxima em função da densidade de degraus.

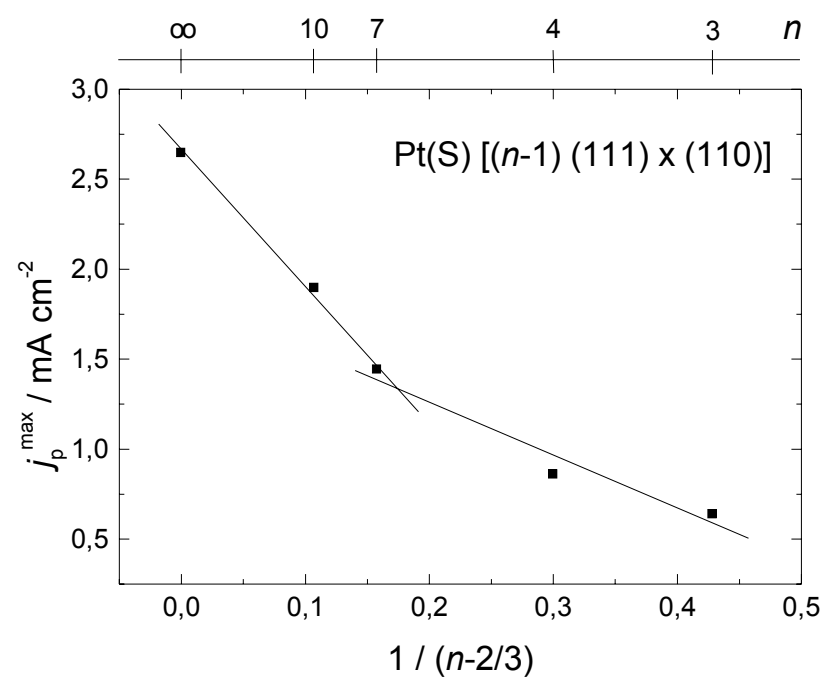

(B)

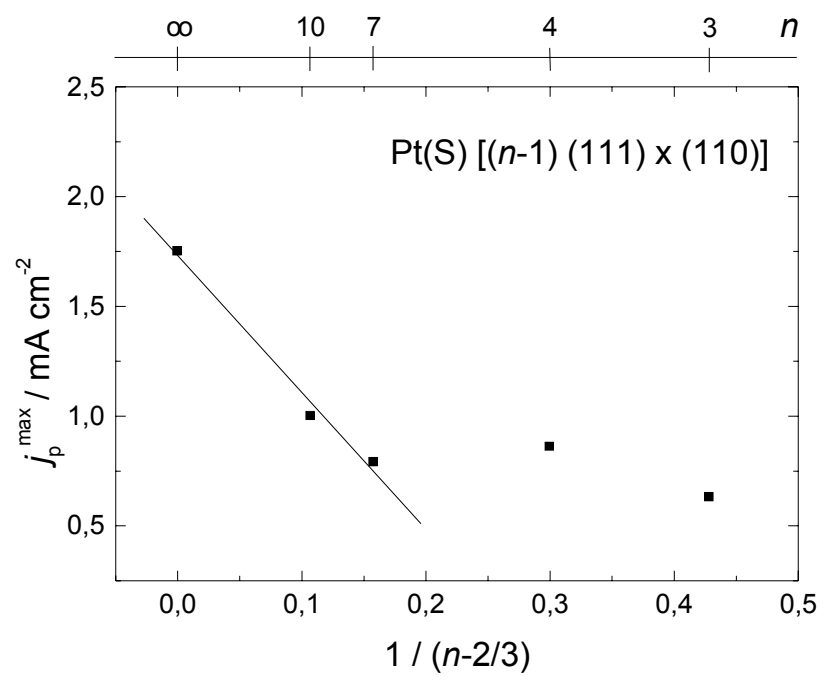

Figura 3. Representação gráfica da densidade de corrente de pico máxima $\left(j_{\mathrm{p}}\right)$ em função da densidade de degraus monoatómicos de orientação (110), para a oxidação electroquímica de (A) D-sorbitol e (B) D-manitol $\left(10^{-2} \mathrm{~mol} \mathrm{dm}^{-3}\right)$; (pico a $\left.0.7 \mathrm{~V}\right)$.

Os valores de densidade de corrente, que apresentam um máximo nesta região, decrescem linearmente com o aumento da densidade de degraus. No entanto, é possível identificar uma alteração do valor do declive para terraços possuindo 
uma dimensão de $\sim 6$ fiadas atómicas (especialmente marcante no caso do Dsorbitol). Tal facto pode estar relacionado com a perda da "característica (111)" nos terraços com dimensão inferior à mencionada, induzindo assim uma alteração na velocidade do processo de oxidação. Este comportamento é semelhante ao observado durante a oxidação electroquímica da glucose no mesmo tipo de superfícies [13].

Série Pt(S) [n (111) x(100)]

As superfícies monocristalinas pertencentes a esta série, designadas genericamente por $\operatorname{Pt}(n+1, n-1, n-1)$, como referido anteriormente, possuem degraus monoatómicos de orientação (100) na junção dos terraços de orientação (111) com a dimensão de $n$ fiadas atómicas.

Os perfis voltamétricos correspondentes à oxidação electroquímica do D-sorbitol nas superfícies do tipo $\operatorname{Pt}(n+1, n-1, n-1)$ são apresentados na Fig. 4. A forma geral dos voltamogramas é muito semelhante à obtida para o voltamograma registado com o eléctrodo $\mathrm{Pt}(111)$ [Ref.].
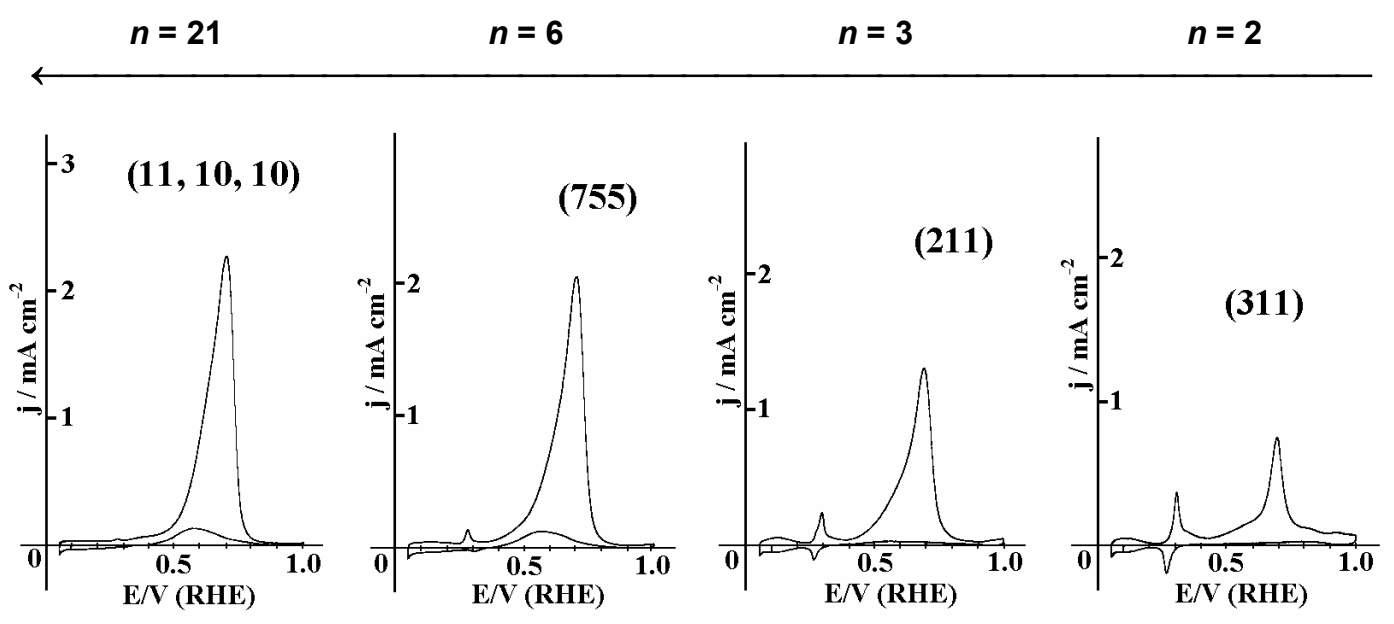

Figura 4. Perfís voltamétricos da oxidação do D-sorbitol $\left(10^{-2} \mathrm{~mol} \mathrm{dm}^{-3}\right)$ em superfícies monocristalinas, com degraus monoatómicos, do tipo $\mathrm{Pt}(\mathrm{S})[n(111) \times(100)]$, em $\mathrm{HClO}_{4}$ $0.1 \mathrm{~mol} \mathrm{dm}^{-3} ; v=50 \mathrm{mV} \mathrm{s}^{-1}$.

A diferença mais significativa reside no desenvolvimento de um pico, localizado $\mathrm{a} \sim 0.3 \mathrm{~V}$, cuja importância aumenta com o aumento do número de degraus de 
orientação (100). Esta segunda contribuição está provavelmente relacionada com a oxidação do D-sorbitol nos degraus de orientação (100), já que esta zona de oxidação apenas ocorre no caso do eléctrodo Pt(100) [7].

A Fig. 5 mostra os perfis voltamétricos para a oxidação de D-manitol em superfícies com degraus monoatómicos, do tipo $\operatorname{Pt}(n+1, n-1, n-1)$, em soluções de $\mathrm{HClO}_{4} 0.1 \mathrm{~mol} \mathrm{dm}{ }^{-3}$.

Na série $\operatorname{Pt}(\mathrm{S})[n(111) \times(100)]$, a presença de degraus (111) orientados conduz a uma modificação do comportamento voltamétrico numa extensão mais apreciável. Novamente, o principal processo de oxidação é bloqueado ao potencial de adsorção de hidrogénio fortemente ligado em terraços (111), mas agora, nos degraus (100), mais explicitamente em superfícies de elevada densidade de degraus, há uma nova contribuição aos potenciais próximos da região de adsorção de hidrogénio numa superfície limpa.

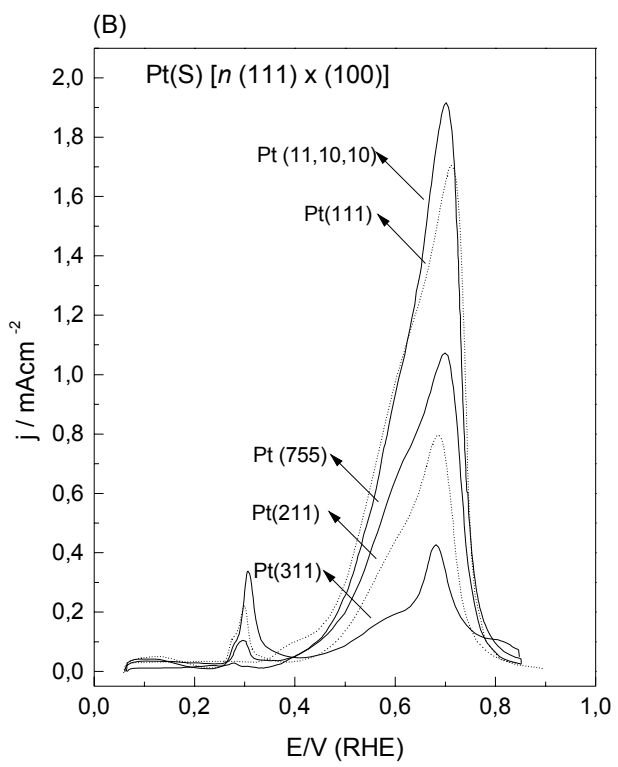

Figura 5. Perfis voltamétricos da oxidação electroquímica do D-manitol $\left(10^{-2} \mathrm{~mol} \mathrm{dm}^{-3}\right)$ em superfícies monocristalinas, com degraus monoatómicos, do tipo $\operatorname{Pt}(\mathrm{S})[n(111) \times$ (100)], em $\mathrm{HClO}_{4} 0.1 \mathrm{~mol} \mathrm{dm}^{-3} ; v=50 \mathrm{mV} \mathrm{s}^{-1}$.

A carga associada a este processo é superior à devida à adsorção de hidrogénio numa superfície limpa (não bloqueada). Para a $\operatorname{Pt}(311)$ este processo é 
comparável, em dimensão, ao pico principal e torna-se o pico mais importante depois do primeiro varrimento (não mostrado nas Figs. 4 e 5). Com a diminuição em $n$, o potencial para este processo cresce gradualmente para $0.30 \mathrm{~V}$, o potencial para a adsorção de hidrogénio fortemente ligado numa superfície $\operatorname{Pt}(100)$. Assim, neste processo, a sua ocorrência a potenciais menos positivos é favorecida por valores baixos de $n$.

(A)

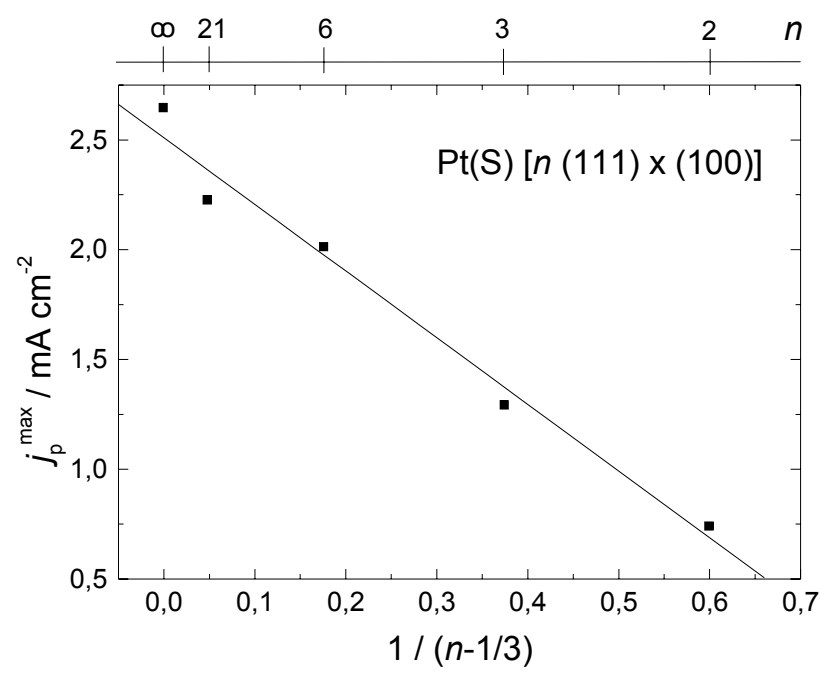

(B)

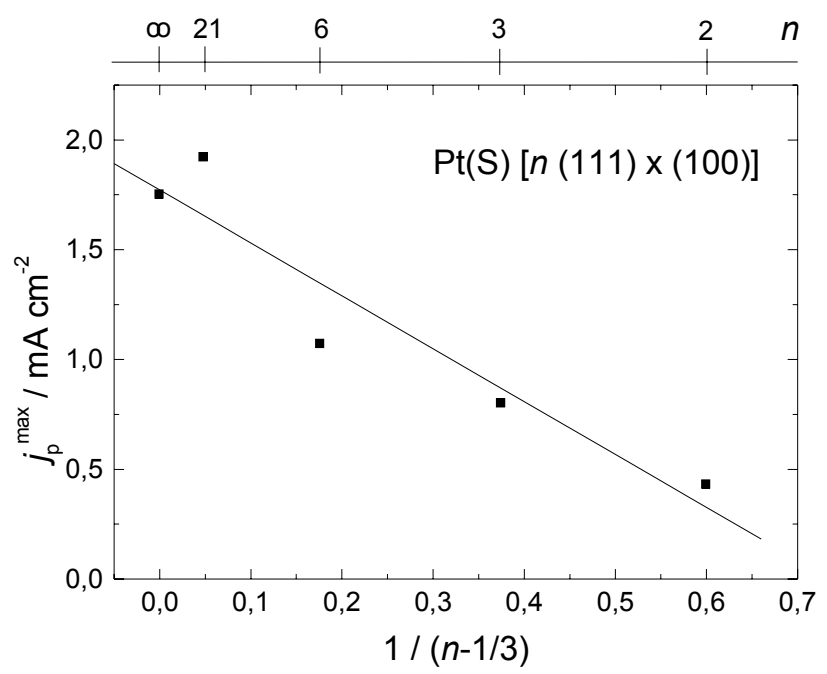

Figura 6. Representação gráfica da densidade de corrente de pico máxima $\left(j_{\mathrm{p}}\right)$ em função da densidade de degraus monoatómicos de orientação (100), para a oxidação electroquímica de (A) D-sorbitol e (B) D-manitol $\left(10^{-2} \mathrm{~mol} \mathrm{dm}^{-3}\right)$; (pico a $\left.0.7 \mathrm{~V}\right)$. 
Em ambas as séries com terraços de orientação (111), $\operatorname{Pt}(\mathrm{S})[(n-1)(111) \times(110)]$ e $\operatorname{Pt}(\mathrm{S})[n(111) \times(100)]$, é preservado o perfil voltamétrico geral de uma superfície (111); porém, degraus do tipo (100) levam a um novo pico a $0.30 \mathrm{~V}$ e degraus de orientação (111) clarificam o ombro no pico principal num pico menor.

A representação gráfica do valor de densidade de corrente máxima em função da densidade de degraus é apresentada na Fig. 6.

A partir desta análise é possível observar que o valor da densidade de corrente máxima, a $0.7 \mathrm{~V}$ (para a oxidação de ambas as moléculas), decresce linearmente com o aumento da densidade de degraus de orientação (100), ou seja, com a diminuição da dimensão dos terraços de orientação (111).

\section{Série Pt(S) [n (100) x (111)]}

As superfícies monocristalinas pertencentes a esta série, designadas genericamente por $\operatorname{Pt}(2 n-1,1,1)$, possuem degraus monoatómicos de orientação (111) e terraços de orientação (100) com a dimensão de $n$ fiadas atómicas.

O comportamento global das superfícies pertencentes a esta série é ilustrado na Fig. 7, onde se apresentam os perfis voltamétricos obtidos para as superfícies (311), (511) e (711).

A título comparativo apresenta-se, na Fig. 7, a resposta voltamétrica do plano basal (100) cuja orientação coincide com a dos terraços nesta série.

A partir da análise das respostas voltamétricas, é possível observar o aparecimento do pico de oxidação característico do plano (100), localizado a $0.75 \mathrm{~V}$, para superfícies com terraços de dimensão superior a 3 fiadas atómicas. Este pico substitui o pico localizado $\mathrm{a} \sim 0.70 \mathrm{~V}$, que pode ser atribuído à oxidação nos "locais" activos de orientação (111).

A Fig. 8 mostra os perfis voltamétricos anódicos para oxidação de D-manitol em superfícies, com degraus monoatómicos, do tipo $\operatorname{Pt}(2 n-1,1,1)$ em soluções de $\mathrm{HClO}_{4} 0.1 \mathrm{~mol} \mathrm{dm}{ }^{-3}$. 
$n=2$

$n=3$

$n=4$
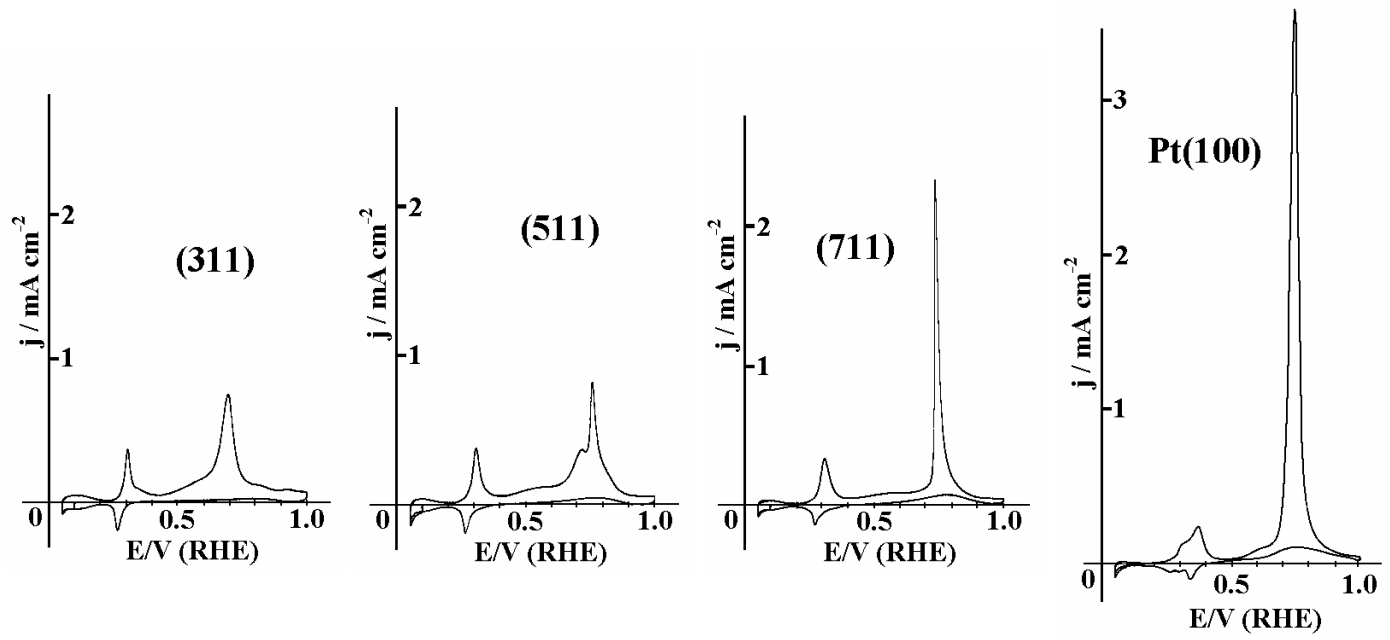

Figura 7. Perfis voltamétricos da oxidação do D-sorbitol $\left(10^{-2} \mathrm{~mol} \mathrm{dm}^{-3}\right)$ em superfícies monocristalinas, com degraus monoatómicos, do tipo $\mathrm{Pt}(\mathrm{S})[n(100) \times(111)]$, em $\mathrm{HClO}_{4}$ $0.1 \mathrm{~mol} \mathrm{dm}^{-3} ; v=50 \mathrm{mV} \mathrm{s}^{-1}$.

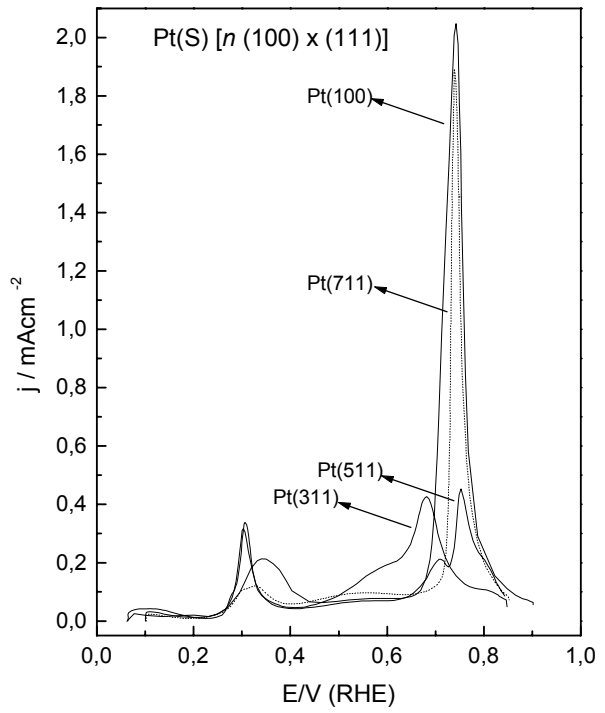

Figura 8. Perfis voltamétricos da oxidação electroquímica do D-manitol $\left(10^{-2} \mathrm{~mol} \mathrm{dm}^{-3}\right)$ em superfícies monocristalinas, com degraus monoatómicos, do tipo $\operatorname{Pt}(\mathrm{S})[n(100) \times$ (111)], em $\mathrm{HClO}_{4} 0.1 \mathrm{~mol} \mathrm{dm}^{-3} ; v=50 \mathrm{mV} \mathrm{s}^{-1}$.

Com degraus orientados (111) num terraço (100) modificações relevantes sobrevêm no comportamento voltamétrico da superfície de baixo índice, $\operatorname{Pt}(100)$. Para a $\operatorname{Pt}(711), n=4$, o perfil é em muito idêntico ao de uma superfície (100) 
ordenada. Contudo, para a próxima superfície com um valor inferior de $n$ o pico estreito a $0.72 \mathrm{~V}$ quase desaparece (a densidade de corrente do pico torna-se $25 \%$ da do pico da $\operatorname{Pt}(711)$ ) e um novo e pequeno pico surge no princípio do pico, cerca de $20 \mathrm{mV}$ antes. Para a $\operatorname{Pt}(311)$ este pico evolui tornando-se o pico principal, com uma desactivação superior à superfície anterior, $\operatorname{Pt}(511)$. Um novo processo inicia-se, a potenciais próximos a $0.30 \mathrm{~V}$, quando a largura nos domínios orientados (100) é igual (ou menos) que três fiadas atómicas. Este processo de oxidação não está presente nem no plano basal (100), nem no plano (111), e parece ser especifico dos domínios (100) estreitos, como já foi notado previamente para a oxidação da glucose nesta série particular de superfícies com degraus $[13,14]$.

A largura dos terraços orientados (100) parece assumir particular importância. Tudo pode significar que para domínios estreitos (100) os polióis não são mais capazes de seguir o mesmo mecanismo de reacção ou que alguma, menos competitiva, forma energética de ligação para um intermediário da reacção, se torna dominante.

Se a densidade de corrente de pico for considerada, a actividade global em relação à oxidação dos dois polióis também decresce, sem excepção, com a introdução de degraus.

Finalmente, há vantagem em salientar que, para a oxidação electroquímica do Dsorbitol e D-manitol em superfícies com degraus monoatómicos, a actividade superficial é observada numa região de potencial mais larga se comparada com os planos basais de platina. Isto é observado também no varrimento inverso (não indicado nas figuras correspondentes) onde há alguma actividade inédita nos planos basais simples (na região de adsorção de hidrogénio, sobretudo).

\section{Considerações finais}

A oxidação electroquímica do D-sorbitol e D-manitol foi estudada para dois conjuntos de superfícies monocristalinas de platina, com degraus monoatómicos, vicinais ao plano basal (111) e ainda para um conjunto de superfícies com degraus monoatómicos vicinais à superfície Pt(100). Este estudo, por voltametria 
cíclica, teve por objectivo obter informação sobre a influência da geometria de sítios superficiais (tanto da orientação dos degraus quanto da dos terraços) no processo de adsorção e na reacção de oxidação dos dois polióis em platina.

A oxidação electroquímica do D-sorbitol e D-manitol em superfícies monocristalinas de platina revela um processo sensível à estrutura superficial. À variação da superfície associam-se diferenças nas densidades de corrente, nos potenciais de pico, nas percentagens de desactivação de densidade de corrente, e no perfil voltamétrico geral para o pico principal e secundário.

Experiências voltamétricas realizadas em três séries de superfícies com degraus, expuseram a sensibilidade da reacção de adsorção e oxidação do D-sorbitol e Dmanitol à simetria dos domínios bidimensionais bem como à distribuição dos defeitos orientados (degraus monoatómicos) nas superfícies de platina. A introdução de defeitos ordenados levou a uma redução geral das densidades de corrente de pico a par de um aumento de actividade (uma menor densidade de corrente) aos potenciais menos positivos. A largura dos terraços é claramente decisiva para o processo de oxidação nas superfícies pertencentes à série $\operatorname{Pt}(\mathrm{S})$ $[(n-1)(111) \times(110)]$.

\section{Agradecimentos}

Este trabalho foi realizado no âmbito de um programa de colaboração científica Portugal/Espanha ("Acción Integrada Hispano-Portuguesa n 1996-6B" e "Acção Integrada Luso-Espanhola n ${ }^{\circ}$ E-18/96"). Agradece-se ainda o apoio financeiro da FCT - CECUL (Unidade de I\&D 301).

\section{Referências}

1. B. Beden, J.-M. Léger, C. Lamy, in: J.O'M. Bockris, R.E. White and B.E. Conway (Eds.), Modern Aspects of Electrochemistry, vol. 22, p. 97, Plenum Press, New York, 1992.

2. R. Adzic, in: J.O’M. Bockris, R.E. White and B.E. Conway (Eds.), Modern Aspects of Electrochemistry, vol. 21, p. 163, Plenum Press, New York, 1991.

3. S.G. Sun, in: J. Lipkowski and P.N. Ross (Eds.), Electrocatalysis, Frontiers of Electrochemistry, vol. 4, p. 243, VCH Publishers, New York, 1998. 
4. J. Clavilier, R. Faure, G. Guinet, R. Durand, J. Electroanal. Chem. 107 (1980) 205.

5. J. Clavilier, D. Armand, S.G. Sun, M. Petit, J. Electroanal. Chem. 205 (1986) 267.

6. A. Rodes, M.A. Zamakhchari, K. El Achi, J. Clavilier, J. Electroanal. Chem. 305 (1991) 115.

7. L. Proença, M.I.S. Lopes, I. Fonseca, A. Rodes, R. Gómez, A. Aldaz, Electrochim. Acta 44 (1998) 1163.

8. A.J. Silva, L. Proença, M.I.S. Lopes, I. Fonseca, A. Rodes, A. Aldaz, Electrochim. Acta 46 (2001) 3147.

9. J. Clavilier, K. El Achi, A. Rodes, Chem. Phys. 141 (1990) 1.

10. A. Rodes, K. El Achi, M.A. Zamakhchari, J. Clavilier, J. Electroanal. Chem. $284(1990) 245$.

11.K. Popovic, A. Tripkovic, N. Markovic, R.R. Adzic, J. Electroanal. Chem. 295 (1990) 79.

12.K. Popovic, A. Tripkovic, N. Markovic, R.R. Adzic, J. Electroanal. Chem. 313 (1991). 181.

13. A. Rodes, M.J. Llorca, J.M. Feliu, J. Clavilier, Anales de Química Int. Ed. 92 (1996) 118.

14. M.J. Llorca, J.M. Feliu, A. Aldaz, J. Clavilier, A. Rodes, J. Electroanal. Chem. 316 (1991) 175. 